LIAMES 2 - pp. 47-60, Primavera 2002

Cristina Messineo

(CONICET- Universidad de Buenos Aires)

\title{
La Marcación Verbal Activa / Inactiva en Toba (Guaycurú) y sus Motivaciones ${ }^{1}$
}

\section{RESUMEN}

En la lengua toba (guaycurú) las categorías pronominales del verbo presentan una marcación diferenciada de caso, ligada al rol semántico de los participantes en el discurso, que distingue entre participantes agentivos o activos y participantes inactivos o pacientivos. Si bien la diferencia entre la marca activa e inactiva se correlaciona con el rol semántico que desempeñan los participantes, el parámetro de la agentividad no parece ser suficiente para establecer tal distinción.

En este artículo se describen los marcadores verbales de persona y se examinan las motivaciones semánticas que subyacen a la diferenciación entre la marcación activa y la inactiva. A modo de introducción, se mencionan algunos rasgos gramaticales que permiten incluir al toba dentro de la categoría de lenguas con sistemas Activos/Inactivos (cf. Seki, 1990, Van Valin, 1990, Mithun, 1991). En segundo lugar, se presentan los marcadores de persona verbal y su relación con las marcas de posesión. Por último, a fin de establecer los parámetros que determinan la marcación pronominal en toba, se analiza su distribución en distintas clases de verbos. Se demostrará que la marcación pronominal activa está determinada no sólo por el grado de control o voluntad que ejercen los participantes de la acción o proceso sino también por la mutabilidad o dinamicidad expresada en el contenido léxico del verbo.

\section{INTRODUCCIÓN}

En este trabajo se describen los marcadores verbales de persona en toba (guaycurú) y se examinan las motivaciones semánticas que subyacen a la diferenciación entre la marcación activa y la inactiva en esta lengua. A fin de establecer los parámetros que determinan dichas marcas, se analiza también la distribución de las mismas en distintas clases de verbos. Se intenta demostrar que la "dinamicidad", la "mutabilidad" y el "cambio de estado" inherentes en la semántica del verbo constituyen los factores principales que determinan la marca activa en toba.

\footnotetext{
${ }^{1}$ Una versión preliminar de este artículo fue presentada al XIII Congreso Internacional de la Asociación de Lingüística y Filología de América Latina (ALFAL), Costa Rica, febrero de 2002. Agradezco especialmente a Ana Gerzenstein por la lectura minuciosa del manuscrito y por sus atinadas sugerencias. Endereço eletrônico: cmessine@mail.retina.ar
} 
Es bien conocido el hecho de que las lenguas del mundo difieren en la marcación de caso. Gran cantidad de descripciones de lenguas indígenas han mostrado la diferencia en que se codifica el sujeto y el objeto directo respecto de la mayoría de las lenguas europeas (Gregores y Suárez, 1967, Seki, 1990, Velázquez Castillo, 1996, Gualdieri, 1998, entre otros).

El toba, una lengua guaycurú hablada por aproximadamente unas 100.000 personas en la región del Gran Chaco (Argentina, Bolivia y Paraguay), presenta muchas de las características de un sistema Activo/Inactivo tal como ha sido descrito por Seki (1990) para el kamaiurá, por Velázquez Castillo (1996) para el guaraní y por Gualdieri (1998) para el mocoví, entre otros. Entre los rasgos que evidencian dicha tipología pueden mencionarse los siguientes: una marcación pronominal escindida que distingue entre participantes activos y participantes inactivos, una división semántica entre verbos activos y estativos, la distinción gramatical entre nombres alienables e inalienables y la ausencia de una clase definida de adjetivos (cf. Messineo, 2000).

Los sistemas gramaticales en los cuales los argumentos de algunos verbos intransitivos son codificados con agentes transitivos y los argumentos de otros con pacientes transitivos han recibido distintas denominaciones: Activo (Klimov, 1979), de Ergatividad Escindida de base semántica (Dixon, 1994) y Split Intransitive (Van Valin, 1990). En dicho sistema gramatical se distinguen los dos roles gramaticales básicos de una construcción transitiva típica: el de Agente (A) - el iniciador o controlador de la actividad- y el de Paciente (P) - el participante más afectado por dicha actividad-. De esta manera, los sujetos activos ya sean transitivos o intransitivos reciben una marca morfológica distinta de la de los sujetos inactivos intransitivos, los cuales a su vez, poseen la misma marca que los objetos de los verbos transitivos. El hecho de que los sujetos intransitivos de los sistemas Activos presenten dos tipos de marca diferenciada, una de ellas isomórfica con las marcas de objeto, como sucede con las lenguas ergativas, ha llevado a algunos autores como Comrie (1978) y Dixon (1994:52 y 70 y ss) a considerar dicho sistema como una variante del sistema ergativo. Dixon (op. cit.: 52,70 y ss) lo denomina de ergatividad escindida [Split ergativity] condicionado por la naturaleza semántica del verbo. Según el autor, estas lenguas emplean una mezcla de estrategias ergativas y acusativas para distinguir verbos con participantes claramente "controladores" de otros con participantes "no controladores o afectados". El lingüista soviético G. Klimov, citado por Seki, (1994), sostiene, sin embargo, que el sistema Activo es un sistema tipológicamente separado del ergativo y que los sistemas Activos / Inactivos se caracterizan por un conjunto de rasgos que se manifiestan en los distintos niveles de la lengua. Entre estos se destaca el hecho de que la estructura lingüística de las lenguas con esa tipología se orienta a la expresión de los roles semánticos de los participantes en el verbo. De esta manera, en los verbos intransitivos se da una identificación entre roles semánticos y relaciones gramaticales que puede esquematizarse de la siguiente manera:

$$
\begin{array}{llll}
\text { Marcación agentiva: } & \mathrm{A} & = & \mathrm{S}_{\mathrm{a}} \\
\text { Marcación pacientiva: } & \mathrm{P} & = & \mathrm{S}_{\mathrm{P}}
\end{array}
$$

La marcación agentiva indica participantes agentes: sujetos de verbos transitivos (A) o intransitivos $\left(\mathrm{S}_{\mathrm{a}}\right.$ ), mientras que la pacientiva codifica participantes pacientes: objetos de verbos transitivos $(\mathrm{P})$ o sujetos de verbos intransitivos $\left(\mathrm{S}_{\mathrm{P}}\right)$. Obsérvense los siguientes ejemplos en toba: 
Messineo: La Marcación Verbal Activa / Inactiva en Toba (Guaycurú) y...

1 A-enseñar D toba 3pos-idioma ${ }^{3}$

"Yo enseño la lengua toba"

(2) mashe sashek

adv 1-Sa- irse

"Ya me voy"

(3)

ayem zasamñi

1 pro $1 \mathbf{S}_{\mathrm{P}}$ - estar cansado

"Estoy cansado"

(4)
so Romualdo
zawaGan
D Romualdo
Z - awa- Gan
"Romualdo me golpeó"
3 A- 1 P - golpear - trans

Existe, por otra parte, una importante discusión acerca de si estas clasificaciones son arbitrarias o motivadas y si poseen base gramatical o semántica. Por un lado, no pueden ser totalmente arbitrarias dado que los verbos pertenecientes a cada clase tienden a compartir ciertas características semánticas. Sin embargo, aunque ciertas clasificaciones coinciden en lenguas no emparentadas genéticamente, en otras sus miembros no pueden predecirse (cf. Mithun, 1991). Por otro lado, algunas descripciones son básicamente sintácticas (Perlmutter, 1978; Burzio, 1981, 1986). Otras, en cambio, tienen base semántica. Van Valin (1990) identifica el "aspecto lexical inherente" (Aktionsart) y la "agentividad" como los dos parámetros semánticos principales que gobiernan la ergatividad escindida, aunque sostiene que las lenguas varían considerablemente respecto de los parámetros que la determinan.

En la siguiente sección, se describen los marcadores de persona y caso en toba.

${ }^{2}$ Para la transcripción fonológica de los ejemplos se utilizan los símbolos que se consignan a continuación:

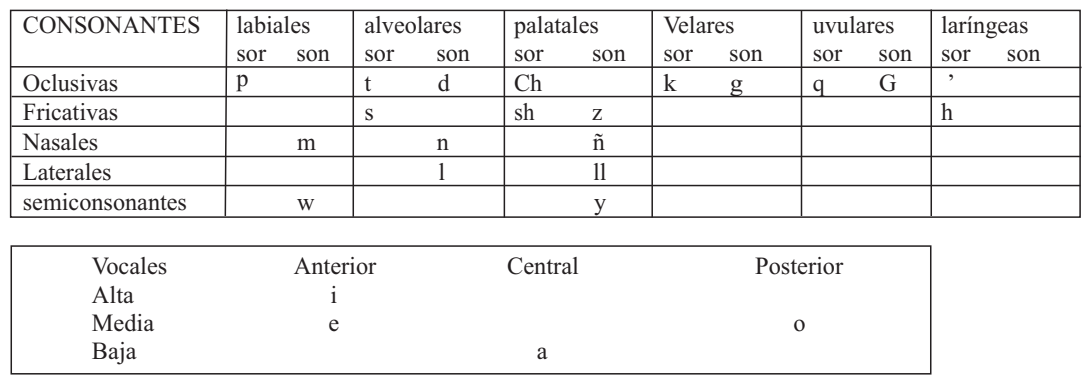

${ }^{3}$ Las siguientes abreviaturas se utilizan para las glosas: A (participante agente); caus (causativo); $\mathrm{Cl}$ (clasificador nominal); D (raíz deíctica); dem (demostrativo); dim (diminutivo); dir (direccional); dur (aspecto durativo); fem (femenino); loc (sufijo locativo); SM (participante medio); P (participante paciente); pl (plural); pos (marcador de poseedor); Pro (pronombre);rec (recíproco); rfl (reflexivo); $\mathrm{S}_{\mathrm{a}}$ (sujeto activo de verbos intransitivos); $S_{p}$ (sujeto paciente de verbos intransitivos); 1 (primera persona); 2 (segunda persona) y 3 (tercera persona). 


\section{MARCADORES DE CASO}

El verbo constituye en toba la clase de palabra morfológicamente más compleja. Las categorías pronominales de persona se prefijan al tema verbal y señalan persona, persona / número (sólo para la segunda plural) y caso semántico. La pluralidad de la primera y de la tercera persona se indica mediante morfemas sufijales; la de la segunda persona, mediante un morfema discontinuo. Otras categorías verbales como el aspecto, la dirección-locación de la acción, las marcas de reflexivo y recíproco y el modo desiderativo se expresan también en la morfología verbal.

Dado que la descripción completa de la morfología del verbo en toba excede los alcances y objetivos de este trabajo, me referiré específicamente al paradigma de pronombres personales dependientes que ocurren como prefijos del tema verbal.

Además de indicar persona y número, las categorías pronominales del verbo presentan una marcación diferenciada de caso, ligada al rol semántico de los participantes en el discurso. Dicha marcas distinguen entre participantes activos o agentivos (controladores o iniciadores del evento expresado por el verbo) y participantes inactivos o pacientivos (afectados por dicho evento). La presencia de un participante medio o semirreflexivo (activo pero afectado por la acción del verbo) también aparece codificada en la morfología pronominal. No obstante, dadas sus características morfosintácticas y semánticas similares a las de la marcación activa, el participante medio no se constituye como una clase independiente. Existen, por lo tanto, dos conjuntos de marcadores de persona: el conjunto I codifica participantes inactivos, objeto de verbos transitivos y pacientes de verbos intransitivos y el conjunto II señala participantes activos, sujeto de verbos transitivos e intransitivos. En el siguiente cuadro se presentan los dos conjuntos de marcadores:

\begin{tabular}{|c|c|c|c|}
\hline & I (Inactivo) & $\begin{array}{l}\text { II (Ac } \\
\text { Activo }\end{array}$ & Activo afectado \\
\hline 1 singular & z- (id-) & s- & ñ- (in-) \\
\hline 1 plural & qad- & s- & ñ- q- \\
\hline 2 singular & 'ad- & 'aw & 'an- \\
\hline $2 \mathrm{pl}$ & qad...i & qaw...i & qan...i \\
\hline 3 & i- /n- & $\begin{array}{l}\mathrm{i}- \\
\mathrm{d}-/ 0-\end{array}$ & n- \\
\hline
\end{tabular}

\section{Cuadro 1}

En las siguientes secciones se presenta un análisis de la morfología pronominal del verbo, de acuerdo con la nómina de marcadores consignados en el cuadro 1.

I. Participante Inactivo

Para la primera persona singular el marcador pronominal que codifica el participante inactivo o paciente es el prefijo /z - / . La segunda persona presenta la marca /'ad-/para el

\footnotetext{
${ }^{4}$ Este prefijo puede ser interpretado como la fusión fonológica del marcador de participante afectado /d-/ y el prefijo de primera persona paciente /i- / que produce la palatalización de la consonate alveolar $(\mathrm{i}+\mathrm{d}=\mathrm{z})$.
} 
singular y /qad-..-i / para el plural. La primera persona plural se indica por medio del prefijo /qad-/ que pareciera ser una extensión de la marca de segunda persona plural. Este conjunto de marcadores es isomórfico con aquél que señala posesión inalienable (cf. Messineo, 2000). El isomorfismo entre la marcación pacientiva de los verbos y la posesiva de los nombres, parece ser también una característica de las lenguas con sistemas Activos.

Obsérvese el contraste entre los siguientes ejemplos, en donde, en la columna de la izquierda la marcación pacientiva señala al sujeto paciente $\left(=\mathrm{S}_{\mathrm{p}}\right)$ de verbos intransitivos, semánticamente inactivos, mientras que en la columna de la derecha, la misma marcación indica el objeto $(=\mathrm{P})$ de verbos activos transitivos:

(6)

(7)

"estar muerto"

a. $\quad$ zi -lew

"me muero"

$1 \mathrm{~S}_{\mathrm{p}}-$ morir

b. 'ad-lew $2 \mathrm{~S}_{\mathrm{p}}$-morir

"te mueres"

-asamñi "estar cansado/a"
z-asamñi "estoy cansado/a
1S S $_{\mathrm{P}}$-estar cansado

b. 'ad-samñi "estás cansado/a" $2 \mathrm{~S}_{\mathrm{P}}$-estar cansado

(8)

a.

b.

a.

b.

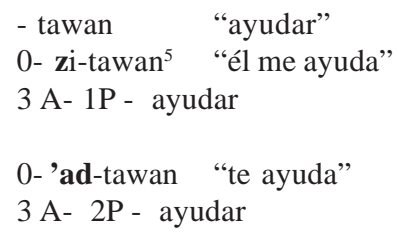

El participante paciente de tercera persona presenta, según sea la clase de verbo, dos patrones de marcación diferenciados:

El sujeto paciente $\left(=\mathrm{S}_{\mathrm{P}}\right)$ de los verbos intransitivos - semánticamente inactivos - se expresa, en algunos casos, por medio del prefijo $/ \mathbf{i}-/^{6}$ :

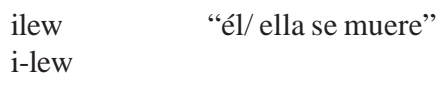

\footnotetext{
${ }^{5}$ Obsérvese que cuando se añade la marca del participante paciente /z/, a los verbos activos transitivos, la marca del agente (/i-/, en este caso), pasa a ser / 0-/.

${ }^{6}$ Nótese que no existe distinción formal entre este marcador y el de la tercera persona activa.
} 
En otros verbos inactivos, el participante de tercera persona aparece marcado por medio del prefijo /n-/, morfema que indica la mayor o menor afectación del participante en la acción. (cf. Participante medio):

(10)

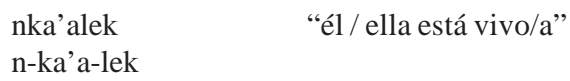

\section{Participante Activo}

\section{a) Agente}

Como puede observarse en los ejemplos siguientes, el marcador /s-/ de primera persona y los marcadores /'aw-/ y /qaw-...-i/ de segunda persona singular y plural respectivamente, codifican el participante agente tanto de verbos transitivos como de intransitivos.

\begin{tabular}{|c|c|c|}
\hline a. & $\begin{array}{l}\text {-alawat } \\
\text { s- alawat } \\
\text { 1A_matar }\end{array}$ & $\begin{array}{l}\text { "matar" } \\
\text { "yo mato" }\end{array}$ \\
\hline b. & $\begin{array}{l}\text { 'aw-alawat } \\
\text { 2A-matar }\end{array}$ & "vos matás" \\
\hline c. & $\begin{array}{l}\text { qaw-alach-i } \\
\text { 2pl A-matar-pl }\end{array}$ & "ustedes matan" \\
\hline a. & $\begin{array}{l}\text {-asot } \\
\text { S- asot } \\
1 S_{\text {a }} \text {-bailar }\end{array}$ & $\begin{array}{l}\text { "bailar" } \\
\text { "yo bailo" }\end{array}$ \\
\hline b. & $\begin{array}{l}\text { 'aw-asot } \\
2 \mathrm{~S}_{\mathrm{a}} \text {-bailar }\end{array}$ & "vos bailás" \\
\hline c. & $\begin{array}{l}\text { qaw-asoch-i } \\
\text { 2plS - bailar-pl }\end{array}$ & "ustedes bailan" \\
\hline
\end{tabular}


Por otra parte, la tercera persona presenta tres marcadores diferentes, según sea el rol semántico del participante y el grado de transitividad expresado por el verbo. En todos los casos, el plural se indica mediante el morfema /d $/ 7$, que ocurre como / $/$ en posición final y como /d / en las demás posiciones:

/-i/ codifica a los participantes activos de verbos transitivos (=A):

\begin{tabular}{|c|c|c|}
\hline (14) & $\begin{array}{l}\text { - alawat } \\
\text { y-alawat } \\
\text { 3A-matar }\end{array}$ & $\begin{array}{l}\text { "matar" } \\
\text { "él mata" }\end{array}$ \\
\hline (15) & $\begin{array}{l}- \text { lo } \\
\text { i- } \quad \text { lo } \\
3 \mathrm{~A}-\text { mirar }\end{array}$ & $\begin{array}{l}\text { "mirar" } \\
\text { "él mira" }\end{array}$ \\
\hline (16) & $\begin{array}{l}\text { - apaGagen } \\
\mathbf{y} \text {-apaGagen } \\
\text { 3A- enseñar }\end{array}$ & $\begin{array}{l}\text { "enseñar" } \\
\text { "él enseña" }\end{array}$ \\
\hline
\end{tabular}

/d-/ ${ }^{8}$ codifica al participante agente de verbos de baja transitividad o típicamente intransitivos $\left(=\mathrm{S}_{\mathrm{a}}\right)$ como se observa en los siguientes ejemplos:

$\begin{array}{ll}\text {-'onaqtaGan } & \text { "trabajar" } \\ \text { do-'onaqtaGan } & \text { "él / ella trabaja" } \\ \text { 3S -trabajar } & \\ \text {-eda } & \text { "moverse" } \\ \text { d-eda } & \text { "él / ella se mueve" } \\ \text { 3S -moverse } & \\ \text {-shiwaGan } & \text { "fumar" } \\ \text { de-shiwaGan } & \text { "él / ella fuma" } \\ \text { S-fumar } & \end{array}$

/0-/ (marcación cero) se presenta en algunos verbos intransitivos, generalmente con participantes inanimados, y fundamentalmente en los verbos locativos:

\footnotetext{
${ }_{8}^{7}$ Esta forma coincide con la marca de poseedor de tercera persona plural de los nombres. ${ }^{8}$ Según Sándalo (citada por Gualdieri, op.cit.:213) existe en kadiweu la marca /d:/ que la autora interpreta como de rol semántico Tema (Afectado). Siguiendo esta hipótesis, Gualdieri encuentra para el mocoví la forma /r-/ ( /d-/) también con la función de marcar un participante paciente que, dependiendo de la clase de verbo, puede o no ser correferente con el sujeto. La misma interpretación puede ser asumida para el toba, en donde /d-/ ocurre en construcciones antipasivas, con verbos de baja transitividad o intransitivos como: deke'e "él/ella come", dewoshi "él/ella cocina".
} 
wahñi

0-wahñi

"él / ella se cae"

$\mathrm{S}_{\mathrm{a}}$-caerse

(21)

pogi

0-pogi

"se cierra"

$3 \mathrm{~S}_{\mathrm{a}}$-cerrarse

(22) wetañi

0-wetañi "está abajo"

$3 \mathrm{~S}_{\mathrm{a}}$ - estar abajo

(23) netalek

0-netalek "está sobre"

$3 \mathrm{~S}$ - estar sobre

Como se observa por lo expuesto, mientras que las personas deícticas (1era y 2da) presentan una única marca morfológica para señalar el participante agente de los verbos activos, en la tercera persona se observa una escisión en dicha marcación que parece estar indicando una gradación en la escala de la transitividad. En efecto, los participantes agentivos de los verbos típicamente transitivos, ocurren con el prefijo/i-/, que representa el grado más alto. En el nivel inmediatamente inferior, se ubican los verbos intransitivos que poseen un participante activo, marcados con el prefijo /d-/. Por último, los verbos locativos con participantes de baja agentividad y algunos verbos intransitivos con participantes inanimados que llevan la marca pronominal /- 0/, ocupan el grado inferior de la escala propuesta. (cf. también Gerzenstein, 1994:137 para el maká).

\section{b) Agente afectado}

La marca pronominal del participante agente afectado es $/ \tilde{\mathbf{n}}-/^{9}$ para la primera persona, /'an-/ y /qan-...-i//10 para la segunda singular y plural respectivamente, y $/ \mathbf{n}-/$ para la tercera.

Dicha marca denota un participante sujeto activo, pero al mismo tiempo semánticamente afectado por la acción del verbo. Esta marcación ha recibido en toba y en otras lenguas guaycurúes distintas interpretaciones. Para el toba, Klein (1978), Buckwalter (1980) y Bigot (1994) la consideran como una forma que amalgama persona y direccionalidad de la acción. Sandalo (1995) para el kadiweo señala la existencia de dos morfemas isomórficos /n-/, uno con el significado de "reflexivo" y el otro de direccional o hither ("hacia aquî"). Por su parte, Grondona (c.p. 1999) considera esa marca en el mocoví también como un direccional (hither).

\footnotetext{
${ }^{9}$ Una posible interpretación del paradigma medio es considerar que el marcador de participante paciente /i-/ (Véase nota 7) se prefija al morfema /n-/ que ocurre en posición inmediatamente anterior a la raíz verbal, resultando entonces: $\mathrm{i}+\mathrm{n}>$ ñ- para la primera persona; Obsérvese que la fusión fonológica del prefijo de primera persona afectada /i-/ con el marcador /n-/, produce la palatalización de la consonante alveolar. Dicha forma alterna con /in-/: inyo "me lavo".

El isomorfismo con el plural de la 1era persona activa constituye un argumento a favor de la interpretación de la marcación media como señalando a un participante activo, pero afectado por la acción del verbo (Véase cuadro 1).
} 
La presencia de una marcación diferenciada para un participante activo pero afectado por la acción del verbo, ha conducido a otros autores (cf. Censabella, 1997 para el toba y Gualdieri, op. cit. para el mocoví) a distinguir entre la voz activa y la voz media en las lenguas guaycurúes. Siguiendo la hipótesis de Kemmer (1993), las autoras mencionadas interpretan que la marcación media constituye el estadio más bajo de una escala que se basa en el grado de diferenciación semántica entre los participantes. Dicha escala incluye también eventos reflexivos y recíprocos. Mientras que los recíprocos involucran a dos participantes correferentes y los reflexivos a un sujeto que actúa sobre sí mismo, en los eventos medios existe una identidad conceptual entre el participante agente y el afectado, siendo el participante activo semánticamente afectado o beneficiado por la acción del verbo. Lo interesante de esta interpretación es que reconoce la existencia de una categoría media ${ }^{11}$ gramaticalizada en la marcación pronominal y basada en el parámetro semántico de diferenciación de los roles de los participantes, propuesta que apoya nuestra hipótesis de considerar el toba dentro de la tipología de las lenguas con sistemas activos. Dicha categoría ocurre codificada por el morfema /n-/ y representa - en relación con la marcación agentiva - un menor grado de separación entre los roles de agente y paciente ${ }^{12}$. En los siguientes ejemplos, en los cuales la misma raíz verbal ocurre con la marca de participante agentivo y la de participante medio, se observa la oposición semántica determinada por el uso de uno u otro marcador. En la columna de la izquierda (a.), la acción expresada en el verbo se presenta dirigida hacia un segundo participante mientras que en la de la derecha (b.), el agente se halla involucrado, se beneficia o es afectado por la acción del verbo y los roles semánticos Agente - Paciente no aparecen claramente diferenciados:

- apaGagen "transmitir conocimiento"

a. $\quad \mathbf{y}$-apaGagen "él enseña"

3A- enseñar

$\begin{array}{lll} & \text { - men } & \text { "intercambiar" } \\ \text { a. } & \begin{array}{l}\text { i- men } \\ \text { 3A-comprar }\end{array} & \\ & & \\ & \text {-él compra" } \\ \text { a. } & \text { y-alawat } & \text { "matar" } \\ & \text { 3A- matar mata" } & \end{array}$

b. n- apaGagen "él aprende" 3M-aprender b. $\quad$ n-men "él vende"
$\quad$ 3M- vender

b. n- alawat-l' at "se suicida" $3 \mathrm{M}$ - matar-rfl ${ }^{13}$

${ }^{11}$ También denominada semirreflexiva: dado que implica a un "participante activo que ejecuta y controla una acción que intimamente lo involucra “. (cf. Mithun 1991: 535)

Esta marca es isomórfica con la marca de posesión alienable (cf. Messineo, 2000), donde la relación entre el poseedor y lo poseído no es tan estrecha como en la posesión inalienable. De la misma manera, la relación entre el Agente y el Paciente de los verbos medios implica un grado mayor de diferenciación de los roles de los participantes que la de los verbos recíprocos y reflexivos. Obsérvense los siguientes ejemplos:

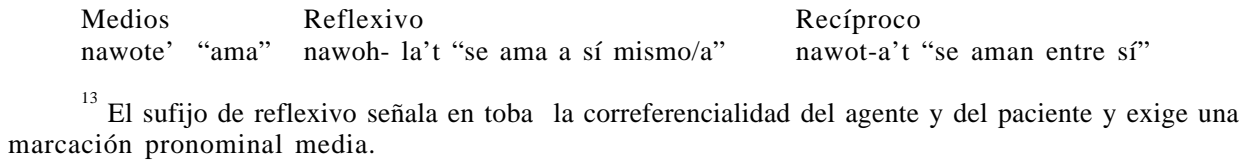




$$
\text { - lo "ver" }
$$
a.
si- lo- shigem "miro para arriba" 1A-ver- dir
b. na- lo- shigem "presto atención" 1SM-ver-dir

Así también se documentaron raíces con los mismos rasgos semánticos pero que pueden llevar tanto la marca de agente afectado como la inactiva. En los siguientes ejemplos, la diferencia entre ambas marcaciones reside en que en la de agente afectado interviene la voluntad, mientras que en la inactiva, no. Esta distinción, no obstante, es sólo perceptible por el participante afectado por la acción:
a.
zasamñi
z-asam- ñi
1-P-cansarse-dir
"Estoy cansado"

b.

ñasamñi
$\tilde{\text { n-asam-ñi }}$ 1M-cansarse-dir

(29)
a. $\quad$ zigelaq
i- $d$ - gelaq
1 - P- regresar
b. ñigelaq
i -n - gelaq
1 -M- regresar

$$
\begin{aligned}
& \text { so'onataGan } \\
& \text { s- 'onataGan } \\
& \text { 1A- trabajar }
\end{aligned}
$$

"Regreso"

"Regreso (por mi propia voluntad)"

En toba, así como en otras lenguas guaycurúes, la marca pronominal media se halla ligada a los siguientes campos semánticos (cf. Kemmer, 1993): cuidados corporales (ntagiñi "se peina"; npikten "se asea"), cambio de postura corporal (nqollin "se agacha"; 'anso'oñi "te sientas"), procesos físicos involuntarios (ñimitchigem "me estremezco"; nsoqchigiñi "transpira"), procesos cognitivos, perceptivos y emocionales (ntowenek "recuerda"; nkoq "se avergüenza"), movimientos direccionales (nwi "llega"; ndo "trae") y acciones realizadas en beneficio propio (ñoqowi "pesco"; ne'epe "él caza").

\section{CLASES DE VERBOS}

Como hemos visto, el caso se distingue en toba mediante la aplicación de prefijos sobre el verbo. El conjunto Inactivo de prefijos (I) se añade a verbos que designan estados y propiedades, mientras que el conjunto Activo (II), a verbos que denotan actividades y acciones. En efecto, según sea el contenido léxico del verbo, la lengua distingue entre dos clases de verbos: inactivos y activos.

La clase de los verbos inactivos contiene un número reducido de verbos (no más de 20) que indican estados, condiciones, o acciones involuntarias e implican participantes experientivos o afectados. Incluye verbos estativos como "estoy vivo" (zika'alek), "me 
muero" (zilew) ${ }^{14}$, "estoy cansado" (zasamñi) así como también verbos descriptivos que denotan cualidades o relaciones y que en otras lenguas podrían ser expresados por medio de adjetivos, como por ejemplo: "es azul" (imalaq), "es blando" (isotak), "soy perezoso" (ziwal). Estos verbos añaden la marca inactiva, aunque también se documentaron algunos ejemplos que pueden recibir ambas marcas. En el siguiente par, el contenido léxico es el mismo pero la diferencia entre las marcas reside en que en (30) no interviene la voluntad, mientras que en (31) se evidencia cierto control de parte del participante involucrado:

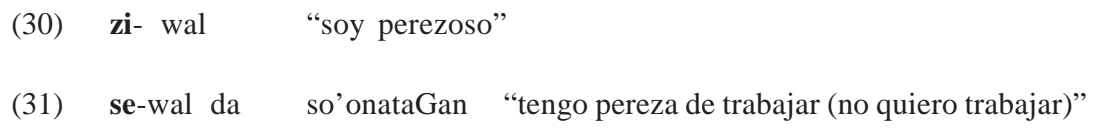

Por otra parte, la clase de los activos incluye, en general, verbos que expresan movimiento de cualquier tipo o actividades, logros y realizaciones (Van Valin, 1990: 222) y que implican agentes semánticos típicos: "jugar" (sa’ashi ), "bailar" (sasot), "enseñar" (sapaGagen), "llegar" (siwi), y "matar" (salawat).

No obstante, en esta clase se integran también otros verbos que denotan participantes con muy bajo grado de voluntad y control o afectados por un cambio involuntario de estado. En este sentido, también añaden la marca activa verbos que expresan las acciones involuntarias de participantes afectados ${ }^{15} \mathrm{o}$ aquellos que implican participantes con muy bajo grado de agentividad. Los participantes pueden ser animados, como en (32), (33) y (34) y (35) o inanimados, como en (36), (37) y (38):

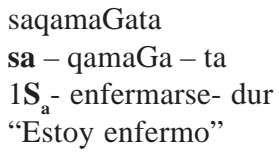

\begin{tabular}{|c|c|c|c|}
\hline \multirow{3}{*}{\multicolumn{2}{|c|}{$\begin{array}{l}\text { demyaqtenaGan } \\
\text { de- myaqten-aGan } \\
\text { 3S - hipar-caus } \\
\text { "Mi hijita tiene hipo" }\end{array}$}} & na & \multirow{3}{*}{$\begin{array}{l}\text { yalole } \\
\text { yal - ole } \\
\text { hijo- dim fem }\end{array}$} \\
\hline & & na & \\
\hline & & $\mathrm{D}$ & \\
\hline am & 'awasoqnata & qalota & \\
\hline Pro 2 & 'aw-soqnata & qalota & \\
\hline vos & $2 \mathbf{S}_{\mathrm{a}}$-toser & mucho & \\
\hline
\end{tabular}

Estás tosiendo mucho

${ }^{14}$ El verbo -lew "morir" fue documentado también con la marcación activa entre las generaciones de hablantes tobas más jóvenes, sin modificación del significado. La alternancia entre ambas marcas en este tipo de verbos es predecible ya que "morir" implica, como "dormir", un cambio de estado.

${ }^{15}$ La distinción entre verbos activos y estativos no es dicotómica y varía de lengua a lengua , dado que forma parte de un continuum generalmente basado en factores culturales En algunas lenguas "vomitar" o "toser" implican acciones involuntarias de participantes afectados, mientras que en otras, para cuyos hablantes son consideradas acciones deseables o de buen gusto, son tratadas como voluntarias y controladas. 


$\begin{array}{llll}\text { ayem } & \text { so'ocheta } & \text { ze } & \text { hawit } \\ \text { Pro } 1 & \text { S - o'oche - ta } & \text { ze } & \text { hawit } \\ \text { yo } & \text { 1S - dormir-dur } & \text { D } & \text { tarde }\end{array}$

"Dormí toda la tarde"

$\begin{array}{lll}\text { demalli } & \text { na } & \text { lchi } \\ \text { de }- \text { malli } & \text { na } & \text { lchi } \\ \text { 3S- hervir } & \text { D } & \text { leche }\end{array}$

"Hierve la leche"

lchi

$\begin{array}{lll}\text { na } & \text { qayaGaray } & \text { ime } \\ \text { na } & \text { qayaGaray } & \mathbf{i}-\text { me } \\ \text { D } & \text { galleta } & \text { 3A-terminarse } \\ \text { "Se terminó la galleta" } & \\ \text { 'iñi } & \text { noyik } & \text { yawik } \\ \text { 'iñi } & \text { noyik } & \text { ya- awik } \\ \text { dem } & \text { casa } & \text { 3A- quemarse } \\ \text { "Se quema esa casa" } & \end{array}$

Así también, los verbos locativos o aquellos en los que el participante agente es una entidad inanimada que simplemente cambia de posición teniendo poco o ningún control sobre el proceso, llevan en toba la marca de participante activo:

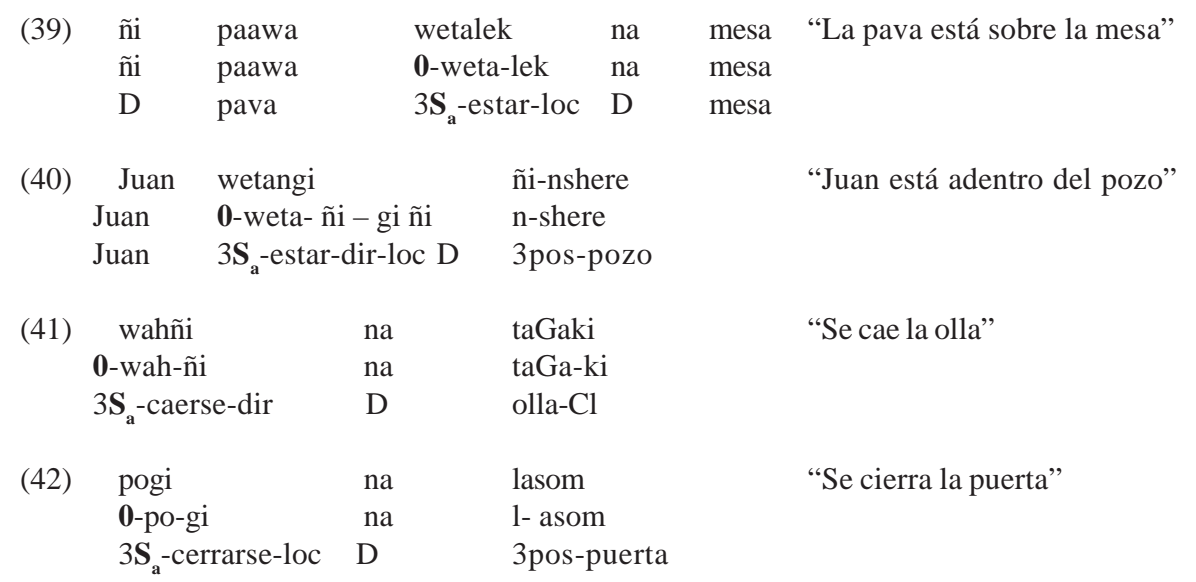

Los ejemplos precedentes muestran que, a diferencia de lo que ocurre con otras lenguas activas / estativas (cf Seki, 1990), el grado de agentividad o el control de los participantes no constituyen en toba rasgos definitorios para determinar la marcación de caso. De hecho, los verbos que implican bajo grado de agentividad o afectados por un cambio involuntario de estado como "dormir", "toser" o "estar en" llevan la marca activa. El factor común que caracteriza a los verbos activos no es, por lo tanto, el control o la volición del participante, sino la naturaleza dinámica del proceso mismo. De esta manera, la dinamicidad o mutabilidad expresada en el contenido léxico del verbo constituye 
también un rasgo fundamental para determinar la marcación activa en esta lengua. De esta manera, el hecho de que los verbos locativos lleven la marca de participante activo puede explicarse considerando que implican un desplazamiento - una actividad previa - del participante hacia determinada locación. (cf. Gerzenstein, 1994:91 para el maká). Así también, verbos no volitivos como "toser" (sasoqnata), "dormir" (so'oche ), "quemarse" (sawik) y "hervir" (semalli), entre otros, implican también cierto dinamismo o cambio en el tiempo, a diferencia de los verbos estativos que denotan permanencia y estabilidad temporal.

\section{CONCLUSIONES}

Los datos analizados en este trabajo muestran que el toba exhibe características de una lengua activa/ estativa, tal como ha sido discutido por diversos autores para otras lenguas indígenas de América del Sur (Seki, 1990, Velázquez Castillo, 1993, Gualdieri, 1998).

Entre los rasgos más importantes se observó que el toba distingue, por un lado, dos conjuntos de marcadores gramaticales de persona: Activo e Inactivo. Desde el punto de vista semántico, la distinción entre los dos primeros se correlaciona con el rol que desempeñan los participantes en el proceso o estado denotado por el verbo: Agente y Paciente. Desde el punto de vista sintáctico, los marcadores Activos codifican sujetos de verbos transitivos $(\mathrm{A})$ o intransitivos $\left(\mathrm{S}_{\mathrm{a}}\right)$, mientras que los Inactivos objetos de verbos transitivos $(\mathrm{P})$ o sujetos de verbos intransitivos $\left(\mathrm{S}_{\mathrm{P}}\right)$.

Por otro lado, existe una división entre verbos activos y estativos basada en la actividad o inactividad expresada en el contenido léxico del verbo.

En el análisis se demostró también que el sistema de marcación de caso está determinado tanto gramatical como semánticamente y que la marca pronominal activa está motivada no sólo por el grado de control o voluntad que ejercen los participantes de la acción o proceso sino también por la mutabilidad (cf. Velázquez Castillo, 1996:13) o dinamicidad expresada en el aspecto lexical del verbo (VanValin, 1990). De esta manera, la agentividad no constituye en toba el rasgo definitorio de esta clase.

En efecto, son varios los factores que interactúan en la lengua para determinar la categorización de caso. Por un lado, la naturaleza del participante involucrado en el proceso: iniciador o controlador vs paciente o afectado. Por otro, las características semánticas inherentes al proceso mismo: dinamicidad o mutabilidad vs inactividad y estabilidad. Ambos parámetros se correlacionan en la mayoría de los casos, pero no en todos, como se observó en los ejemplos (32) a (42), en los cuales la marcación activa denota procesos pero participantes no iniciadores o carentes de control. En otros casos, como los consignados en (30) y (31) la naturaleza del participante puede por sí sola determinar la marca.

En síntesis, siguiendo a Velázquez Castillo (op.cit.: 18), podemos afirmar que el concepto de actividad constituye en toba el resultado de la interacción entre un participante que inicia, ejecuta, instiga o controla la acción y la dinamicidad inherente al contenido léxico del verbo. Dichos factores coocurren en los casos prototípicos, pero la presencia de ambos no es necesaria para determinar si una forma es activa o inactiva. 


\section{REFERENCIAS BIBLIOGRÁFICAS}

BIGOT, M. (1998). La lengua qom (toba) del Chaco (Argentina). Expresión del espacio en los lexemas verbales. Actas de las II Jornadas de Lingüística Aborigen, pp. 177-189. Buenos Aires: F. F y L (UBA).

BUCKWALTER, A. (1980). Vocabulario Toba. Buenos Aires: Talleres Grancharoff.

BURZIO, L., (1981). Intransitive verbs and Italian auxiliares. Cambridge, MA: MIT Dissertation. .(1986). Italian Syntax: A Government-Binding Approach. Dordrecht: Reidel

CENSABELlA, M. (1997). Gramática funcional de la lengua toba. Sincronía dinámica de una lengua amenazada (Inédito).

COMRIE, B. (1978). Aspect (2da ed.) Cambridge: Cambridge University. Press.

DIXON, R. (1994). Ergativity. Cambridge Studies in Linguistics 69. Cambridge: Cambridge University. Press.

GERZENSTEIN, A. (1994). El maká. Estudio descriptivo. Archivo de Lenguas Indoamericanas. Serie "Nuestra América". Buenos Aires: FFyL (UBA).

GREGORES, E. y J. SUÁREZ (1967). A description of colloquial Guaraní. The Hague: Mouton.

GUALDIERI, B. (1998). Mocoví (Guaycurú). Fonologiae e morfossintaxe. Tesis Doctoral. Campinas, São Paulo: Universidade Estadual de Campinas.

KEMMER, S. (1993). The Midlle Voice. Amsterdam/ Philadelphia: J. Benjamins.

KLEIN, H. (1978) Una gramática de la lengua toba: morfología verbal y nominal. Montevideo: Universidad. de la República (ed. en inglés 1974).

KLIMOV, G. A. (1979). On the position of the ergative type in typological classification. En F. Plank (ed) Ergativity. Towards as Theory of Grammatical Relations, pp. 327-332. London: Academic Press.

MESSINEO, C. (2000). Estudio del toba hablado en la provincia del Chaco (Argentina). Aspectos gramaticales y discursivos. Tesis Doctoral, FFyL, UBA.

MITHUN, M.. (1991). Active/ agent case marking and its motivation. Language 67 (3):510-46.

PERLMUTTER, D.(1978). Impersonal passives and the unacussative hypotesis. Berkeley Linguistics Society 4. 157-89.

SANDALO, F. (1995). “A grammar of kadiwéo". Ph D. Dissertation. University. of Pittsburg

SEKI, L. (1990). Kamaiurá (Tupi-Guaraní) as an active-stative language. En D. Payne (ed) Amazonian Linguistics: Studies in Lowland South american Languages, 367-92. Austin: University of Texas Press.

VAN VALIN, R. (1990). Semantic Parameters of split intransitivity. Language, 66 (2): 221-260.

VELAZQUEZ CASTILlO, M. (1996). The Grammar of Possesion: Inalienability, Incorporation and Possessor Ascension in Guaraní. Amsterdam/ Philadelphia: J. Benjamins. 